\title{
Relativistic electron energy loss and induced radiation emission in two-dimensional metallic photonic crystals I: formalism and surface plasmon polariton
}

\author{
Tetsuyuki Ochiai and Kazuo Ohtaka \\ Center for Frontier Science, Chiba University, Chiba 263-8522, Japan
}

(Dated: October 31, 2018)

\begin{abstract}
A fully relativistic description of the electron energy loss and the induced radiation emission in arbitrary arrays of non-overlapping metallic cylinders is presented in terms of the multiple scattering method on the basis of vector cylindrical waves. Numerical analysis is given for dilute and dense arrays of Aluminum cylinders with a nanoscale diameter. The results of the electron energy loss spectrum are well correlated with the dispersion relation of coupled surface plasmon polaritons, and can be interpreted with an effective medium approximation when the electron runs inside the arrays. In addition, the cavity modes localized in the grooves between the cylinders can affect strongly the electron energy loss spectrum.
\end{abstract}

\section{INTRODUCTION}

Recently, much interest has been attracted in optical properties of composite materials such as cluster of metallic nano-particles 1 , photonic crystal ${ }^{2.3}$, and lefthanded material ${ }^{4.5 .6}$. They have the potential ability to enhance various optical processes with their rich spectrum, and will be a key component of future optoelectronics devices. So far, their properties have been investigated mainly with far-field optical equipment. However, near-field responses of them are of great importance, because much information can be attained from the evanescent light involved in composite materials.

The Electron energy loss (EEL) spectroscopy in a scanning transmission electron microscope is a useful tool to investigate both surface and bulk excitations of samples. In a typical EEL experiment the kinetic energy of electron is on the order of $100[\mathrm{keV}]$, and a low-energy part of the EEL spectrum is related to a collective excitation such as surface plasmon. Thus, the spectrum is well interpreted in terms of a classical macroscopic theory on the basis of an effective dielectric function, taking account of geometry and anisotropy of samples. For instance, the EEL of a multi-wall fullerene can be well explained with an effective medium in which the dielectric constant of the normal direction to the multi-walls is different from that of the other direction ${ }^{7}$

There are extensive references on the EEL spectrum for various geometrical objects ${ }^{8.9,10,11}$, though most works neglect the retardation effect. As for the composite materials, Maxwell-Garnett(MG)-type approximations have been widely used. In the last decade a remarkable progress has been made on this subject. Pendry et al. proposed a new theoretical method for the EEL of periodically arranged nano-structures 12 in terms of the real-space transfer matrix of the electro-magnetic wave ${ }^{13}$ García de Abajo et al. developed a multiplescattering method for clusters of nano-particles, on the basis of vector spherical waves ${ }^{14.15}$ and of the boundary element method ${ }^{16.17}$ These methods are very powerful and in principle can be adapted to various complex geometries. However, owing to the spatial discretization used in the transfer matrix method and in the boundary element method, the accuracy of the results reduces to some extent at high frequencies.

Here, we present a fully relativistic multiple-scattering approach focused on arrays of non-overlapping cylinders by using vector cylindrical waves. Though at present the method works only for the cylinders with isotropic dielectric functions, in principle it can work for anisotropic cylinders such as multi-wall carbon nano-tubes $\frac{18}{}$. With this method the light scattering, as well as the EEL and the induced radiation emission in arbitrary arrays of cylinders can be treated in a unified manner by solving exactly the multiple-scattering equation. In addition, since the method utilizes the Fourier decomposition along the cylindrical axis, the local optical responses of a certain wave vector along the axis can be obtained. This property is feasible in order to see the effects of the localized electro-magnetic modes of cylinders in detail.

A certain amount of the EEL in composite materials is caused by the induced radiation emission. As for a metallic nano-particle, the phenomena is known as the surface plasmon radiation emission 19 By arranging nano-particles periodically, so-called Smith-Purcell(SP) radiation ${ }^{20.21}$ takes place. One of the authors (K.O.) and his collaborators have studied the SP radiation in the photonic crystals composed of dielectric spheres in detail, where a notable enhancement of the intensity of the SP radiation takes place owing to the singular state density of photon in the photonic crystals ${ }^{22.23 .24}$ However, to the best of our knowledge, none has been reported concerning the radiation emission from arrays of cylinders. Thus, the quantitative evaluation of the radiation emission in the arrays is another theme of the paper.

On the other hand, the determination of the effective dielectric function of composite materials is still valuable at low frequencies. It gives a concise explanation of the absorption spectrum as well as the EEL spectrum of the composite materials in bulk ${ }^{25}$ Moreover, the determination is an important issue in the field of left-handed material, which has negative permittivity and permeability simultaneously. Several methods to determine them have been proposed by many authors ${ }^{26.27 .28}$ Here, we propose 
an alternative method by using the scattering matrix of semi-infinite photonic crystals and compare the EEL in the effective medium with that of photonic crystal.

This paper deals mainly with the formalism of our method as well as the numerical analysis on clusters of Aluminum cylinders with a diameter of a few nanometers, bearing carbon nano-tube arrays in mind. In the paper II we will deal with a metallic photonic crystal whose lattice constant is comparable with or larger than the plasma wavelength of the constituent metallic cylinders. Since it is very bulky to present a comprehensive analysis on the EEL and the induced radiation emission in a single paper, we should discuss the above topics separately. In a nano-structure, which is discussed in this paper, the relevant range in wavelength, which is near the surface plasma wavelength of metal, is much larger than the diameter and than the pitch of the structure. As a result, effects of usual photonic bands, which come from zone foldings and degeneracy shifts on Bragg planes, do not appear in the EEL spectrum and the induced radiation emission spectrum at relevant frequencies. In particular there is no remarkable feature in the SP radiation emission spectrum. Instead, unusual photonic bands of coupled surface plasmon polaritons have a strong influence on the EEL spectrum at very low frequencies. Moreover, in the relevant frequency range an effective medium approximation can be reasonably adapted to the structure. As for the structure which will be discussed in the paper II, we will show that effects of both the usual photonic bands are very pronounced for the EEL and the induced radiation emission spectra.

The paper is organized as follows. In Sec.II we briefly summarize the vector cylindrical wave formalism. Using the formalism the dispersion relation of the surface plasmon polariton in an isolated metallic cylinder is obtained. The multiple scattering method is adapted to the EEL and the induced radiation emission in clusters of metallic cylinders in Sec.III. In Sec. IV the expression of the EEL in metallic photonic crystals is derived. The numerical results of the EEL spectrum are compared both with those of the isolated cylinder and with those of an effective homogeneous medium. Finally, we summarize the results.

\section{SURFACE PLASMON POLARITON IN AN ISOLATED CYLINDER}

An infinitely long metallic cylinder with a circular cross section can support an electro-magnetic surface-localized mode that is called surface plasmon polariton(SPP). The SPP is characterized by an angular momentum $l$, a wave number $k_{z}$, and an angular frequency $\omega$, owing to the ro- tation invariance with respect to the cylindrical axis, the translational invariance along the axis, and the translational invariance of time, respectively. Before considering its dispersion relation, it is valuable to note some formulas of the light scattering by an isolated cylinder using vector cylindrical waves. ${ }^{29}$ From now on, we take the cylindrical axis to the z-axis. Assume that a monotonic incident wave with a momentum $k_{z}$ along the cylindrical axis is scattered by an isolated cylinder with a dielectric function $\varepsilon_{a}(\omega)$ and a radius $r$ embedded in a host with a permittivity $\varepsilon_{b}$. Throughout the paper $\varepsilon_{b}$ is taken to be 1 in numerical calculations, though we keep it unspecified in equations. The incident wave can be written as a superposition of vector cylindrical waves:

$$
\begin{array}{r}
\mathbf{E}^{0}(\mathbf{x})=e^{i k_{z} z}\left[\left(-\frac{1}{\lambda_{b}} \hat{z} \times \nabla_{\|}\right) \psi^{M, 0}(\mathbf{x})\right. \\
\left.+\left(\frac{i k_{z}}{\lambda_{b} q_{b}} \nabla_{\|}+\frac{\lambda_{b}}{q_{b}} \hat{z}\right) \psi^{N, 0}(\mathbf{x})\right], \\
\psi^{\beta, 0}(\mathbf{x})=\sum_{l} J_{l}\left(\lambda_{b} \rho\right) e^{i l \theta} \psi_{l}^{\beta, 0} \quad(\beta=M, N), \\
\lambda_{b}=\sqrt{q_{b}^{2}-k_{z}^{2}}, \quad q_{b}=\sqrt{\varepsilon_{b}} \frac{\omega}{c}
\end{array}
$$

where $c$ is the speed of light in vacuum, $(\rho, \theta, z)$ is the cylindrical coordinate, $\hat{z}$ is the unit vector along the $z$ direction, and $\nabla_{\|}$is the differential operator with respect to $(\rho, \theta)$. Throughout the paper, we take the following convention of the square root of a complex number: $\operatorname{Im} \sqrt{w} \geq 0$ for $\operatorname{Im}(w) \geq 0$. Sometimes, we call the $\mathrm{M}(\mathrm{N})$ field the $\mathrm{P}(\mathrm{S})$ polarization. At $k_{z}=0$ the $\mathrm{M}(\mathrm{N})$-field corresponds to the TE(TM) polarization.

A metallic or dielectric cylinder scatters light irrespective of whether the light is evanescent or not. By imposing the boundary condition of Maxwell's equation, we can solve the scattering problem exactly. The induced wave scattered by the cylinder is given by

$$
\begin{aligned}
& \mathbf{E}^{\text {ind }}(\mathbf{x})=e^{i k_{z} z}\left[\left(-\frac{1}{\lambda_{b}} \hat{z} \times \nabla\right) \psi^{M, \text { ind }}(\mathbf{x})\right. \\
& \left.+\left(\frac{i k_{z}}{\lambda_{b} q_{b}} \nabla_{\|}+\frac{\lambda_{b}}{q_{b}} \hat{z}\right) \psi^{N, \text { ind }}(\mathbf{x})\right] \text {, } \\
& \psi^{\beta, \text { ind }}(\mathbf{x})=\sum_{l} H_{l}\left(\lambda_{b} \rho\right) e^{i l \theta} \psi_{l}^{\beta, \text { ind }} \text {, } \\
& \psi_{l}^{\beta, \text { ind }}=\sum_{\beta^{\prime}} t_{l}^{\beta \beta^{\prime}} \psi_{l}^{\beta, 0}
\end{aligned}
$$

Here, $H_{l}$ is the Hankel function of first kind and $t_{l}^{\beta \beta^{\prime}}$ is the $t$-matrix of the cylinder, its analytical expression being 


$$
\begin{aligned}
& \left(\begin{array}{ll}
t_{l}^{M M} & t_{l}^{M N} \\
t_{l}^{N M} & t_{l}^{N N}
\end{array}\right)=-\left(\begin{array}{ll}
d_{l}^{>M M} & d_{l}^{>M N} \\
d_{l}^{>N M} & d_{l}^{>N N}
\end{array}\right)\left(\begin{array}{ll}
d_{l}^{<M M} & d_{l}^{<M N} \\
d_{l}^{<N M} & d_{l}^{<N N}
\end{array}\right)^{-1} \\
& \left(\begin{array}{ll}
d_{l}^{>} M M & d_{l}^{>} M N \\
d_{l}^{>N M} & d_{l}^{>N N}
\end{array}\right)=\left(\begin{array}{cc}
\rho_{b} J_{l}^{\prime}\left(\rho_{a}\right) J_{l}\left(\rho_{b}\right)-\rho_{a} J_{l}\left(\rho_{a}\right) J_{l}^{\prime}\left(\rho_{b}\right) & l \frac{k_{z}}{q_{a}}\left(\frac{\rho_{b}}{\rho_{a}}-\frac{\rho_{b}}{\rho_{a}}\right) J_{l}\left(\rho_{a}\right) J_{l}\left(\rho_{b}\right) \\
l \frac{k_{z}}{q_{b}}\left(\frac{\rho_{b}}{\rho_{a}}-\frac{\rho_{b}}{\rho_{a}}\right) J_{l}\left(\rho_{a}\right) J_{l}\left(\rho_{b}\right) & \frac{q_{a}}{q_{b}} \rho_{b} J_{l}^{\prime}\left(\rho_{a}\right) J_{l}\left(\rho_{b}\right)-\frac{q_{b}}{q_{a}} \rho_{a} J_{l}\left(\rho_{a}\right) J_{l}^{\prime}\left(\rho_{b}\right)
\end{array}\right), \\
& \left(\begin{array}{ll}
d_{l}^{<M M} & d_{l}^{<M N} \\
d_{l}^{<N M} & d_{l}^{<N N}
\end{array}\right)=\left(\begin{array}{cc}
\rho_{b} J_{l}^{\prime}\left(\rho_{a}\right) H_{l}\left(\rho_{b}\right)-\rho_{a} J_{l}\left(\rho_{a}\right) H_{l}^{\prime}\left(\rho_{b}\right) & l \frac{k_{z}}{q_{a}}\left(\frac{\rho_{b}}{\rho_{a}}-\frac{\rho_{b}}{\rho_{a}}\right) J_{l}\left(\rho_{a}\right) H_{l}\left(\rho_{b}\right) \\
l \frac{k_{z}}{q_{b}}\left(\frac{\rho_{b}}{\rho_{a}}-\frac{\rho_{b}}{\rho_{a}}\right) J_{l}\left(\rho_{a}\right) H_{l}\left(\rho_{b}\right) & \frac{q_{a}}{q_{b}} \rho_{b} J_{l}^{\prime}\left(\rho_{a}\right) H_{l}\left(\rho_{b}\right)-\frac{q_{b}}{q_{a}} \rho_{a} J_{l}\left(\rho_{a}\right) H_{l}^{\prime}\left(\rho_{b}\right)
\end{array}\right), \\
& \rho_{i}=\lambda_{i} r \quad(i=a, b), \quad \lambda_{a}=\sqrt{q_{a}^{2}-k_{z}^{2}}, \quad q_{a}=\sqrt{\varepsilon_{a}} \frac{\omega}{c}
\end{aligned}
$$

It should be emphasized that except for $k_{z}=0$ or $l=0$, the $\mathrm{M}(\mathrm{N})$ polarization mixes with the $\mathrm{N}(\mathrm{M})$ polarization in the induced wave. This property is quite distinct from the case of an isolated sphere, where the polarization mixing does not take place. In the light scattering by cylinder there is another distinct point from that by sphere. Since $\lambda_{b}$ is the wave number in the $(x, y)$ plane, the induced wave is evanescent when $\lambda_{b}$ is pure imaginary. On the other hand the induced wave by the sphere behaves as $h_{l}\left(q_{b}|\mathbf{x}|\right)$, where $h_{l}$ is the spherical Hankel function of first kind. This means that induced wave by the sphere is always real and has a net flux at $|\mathbf{x}|=\infty$ irrespective of whether the incident wave is evanescent or not.

Next, we focus on the SPP mode in an isolated metallic cylinder. The SPP mode is a real eigenmode in the cylinder and can exist without the incident light. Therefore, taking account of Eq. (6) the equation that determines the dispersion relation of the SPP is given by

$$
\left(\begin{array}{cc}
t_{l}^{<M M} & t_{l}^{<M N} \\
t_{l}^{<N M} & t_{l}^{<N N}
\end{array}\right)^{-1}\left(\begin{array}{c}
\psi_{l}^{M, \text { ind }} \\
\psi_{l}^{N, \text { ind }}
\end{array}\right)=0
$$

Like as the SPP on a flat metal/air interface, whose dispersion relation is given by the pole of the interface Smatrix between the metal and air, the dispersion relation of the SPP in a metallic cylinder is given by the pole of the t-matrix. The above equation leads to the following secular equation:

$$
\operatorname{det}\left(\begin{array}{cc}
d_{l}^{<M M} & d_{l}^{<M N} \\
d_{l}^{<N M} & d_{l}^{<N N}
\end{array}\right)=0
$$

for the SPP mode with angular momentum $l$. Strictly speaking, we must say that the SPP mode is referred to the mode with pure imaginary $\lambda_{a}$, when the cylinder is of metal. The secular equation also determines the dispersion relation of the guided modes, in which $\lambda_{a}$ is real and positive, when the cylinder is of dielectric.

For simplicity, from now on, we restrict our consideration to an Aluminum cylinder with diameter 2.5[nm], though the formalism presented in the paper can be adapted to any types of cylinders as long as an isotropic dielectric function of the cylinder is concerned. The dielectric function of the Aluminum can be approximated

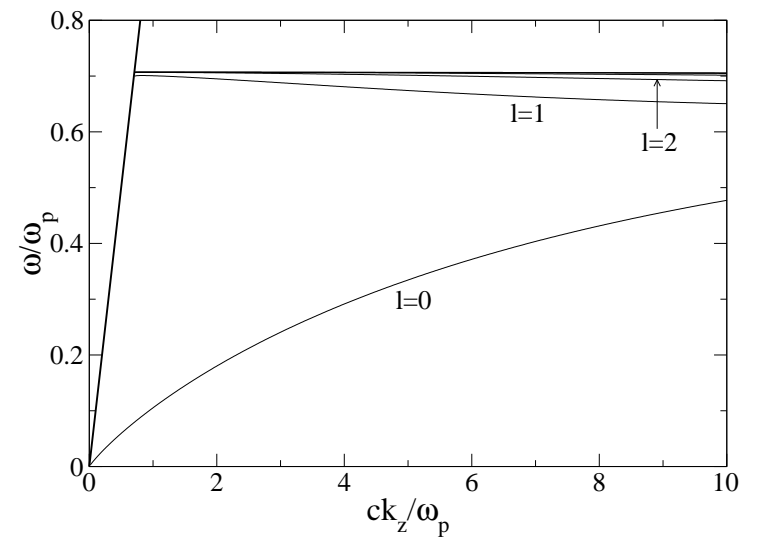

FIG. 1: The dispersion relation of the surface plasmon polariton of the Aluminum cylinder with diameter $2.5[\mathrm{~nm}]$ in air. The loss-less Drude dielectric function with $\hbar \omega_{p}=15[\mathrm{eV}]$ was assumed for the Aluminum cylinder. The bold line is the light line.

well with the Drude formula:

$$
\varepsilon_{a}(\omega)=1-\frac{\omega_{p}^{2}}{\omega(\omega+i \eta)},
$$

$\hbar \omega_{p}$ and $\hbar \eta$ being $15[\mathrm{eV}]$ and $1[\mathrm{eV}]$, respectively. We focus on this cylinder embedded in air $\left(\varepsilon_{b}=1\right)$ in numerical calculations of the paper. The small diameter of the cylinder is comparable with that of a carbon nano-tube and the plasma frequency is $3.67 \times 10^{15}[\mathrm{~Hz}]$ and $82.7[\mathrm{~nm}]$ in terms of wavelength, which corresponds to ultra-violet light. Though the cylinder has a nano-scale diameter, it seems still reasonable to apply the macroscopic dielectric function to the cylinder.

In Fig. 1 the dispersion relation of the SPP modes of the Aluminum cylinder is shown. In this case the SPP mode with $l=0$, which is the solution of $d_{0}^{<N N}=0$, is quite distinct from those with $l \geq 1$. The mode with $l=0$ ends at $\omega=0$ and has positive slope in the $\left(\omega, k_{z}\right)$ space, whereas the other modes end at finite $\omega$ on the light line and have negative slopes. As $l$ increases, the dispersion curves approach to $\omega=\omega_{p} / \sqrt{2}$. Though the dispersion curves end on the light line, they can be extrapolated inside the light-cone. With this extrapolation 
we can understand how the SPP resonance occurs when real light is incident on the cylinder. The resonance also affects significantly the induced radiation emission when a charged particle passes near the cylinder.

\section{ELECTRON ENERGY LOSS IN CLUSTER OF CYLINDERS}

The formalism presented above serves to describe the EEL and the induced radiation emission when a charged particle runs near a cluster of cylinders. In this method the retardation effect is fully taken into account, though the recoil of the charged particle is neglected. In a typical EEL experiment the kinetic energy of the electron is about $100[\mathrm{keV}]$, whereas the total energy loss is less than $0.01 \%$ of it, so that the assumption of neglecting the recoil is fairly justified.

Let us consider a charged point particle with a charge $e$ and a velocity $v$ runs near the cluster composed of nonoverlapping metallic cylinders aligned in the $z$-direction, whose positions are specified by two-dimensional ( $x$ and $y$ ) vector $\mathbf{x}_{\alpha}(\alpha=1,2, \ldots, N), N$ being the number of the cylinders. For simplicity, we assume that the charged particle does not penetrate any cylinders in the cluster and the trajectory of the particle is perpendicular to the cylindrical axes. Therefore, without losing generality, the position of the particle at time $t$ is taken to $\mathbf{x}_{t}=\left(v t, y_{0}, z_{0}\right)$ in the Cartesian coordinate. As is known well, a running charged particle is accompanied by the electro-magnetic wave that is given by

$$
\begin{aligned}
& \mathbf{E}^{0}(\mathbf{x}, \omega)=-\frac{\omega \mu_{0} e}{2} \int \frac{d k_{z}}{2 \pi} \epsilon^{ \pm} e^{i \frac{\omega}{v} x+i \gamma\left|y-y_{0}\right|+i k_{z}(z-z(0)}, \\
& \epsilon^{ \pm}=\left(\begin{array}{c}
\frac{1}{\gamma}\left(1-\frac{1}{q_{b}^{2}}\left(\frac{\omega}{v}\right)^{2}\right) \\
\mp \frac{1}{q_{b}^{2}} \frac{\omega}{v} \\
-\frac{1}{q_{b}^{2}} \frac{\omega}{v} \frac{k_{z}}{\gamma}
\end{array}\right) \\
& \gamma=\sqrt{q_{b}^{2}-\left(\frac{\omega}{v}\right)^{2}-k_{z}^{2}}
\end{aligned}
$$

in the time-Fourier component. Here, the superscript of $\epsilon$ is referred to the sign of $y-y_{0}$. If the light velocity in the background medium is smaller than $v$, the electromagnetic wave becomes real, yielding the Cerenkov radiation. Here, we restrict ourselves to the region $v<c / \sqrt{\varepsilon_{b}}$, so that the electro-magnetic wave is evanescent $(\gamma$ is pure imaginary).

The above expression can be transformed into a linear combination of the vector cylindrical waves centered at $\mathbf{x}=\mathbf{x}_{\alpha}$ as

$$
\begin{gathered}
\mathbf{E}^{0}(\mathbf{x}, \omega)=\int \frac{d k_{z}}{2 \pi} e^{i k_{z}\left(z-z_{0}\right)}\left[\left(-\frac{1}{\lambda_{b}} \hat{z} \times \nabla\right) \psi_{\alpha}^{M, 0}(\mathbf{x})\right. \\
\left.+\left(\frac{i k_{z}}{\lambda_{b} q_{b}} \nabla_{\|}+\frac{\lambda_{b}}{q_{b}} \hat{z}\right) \psi_{\alpha}^{N, 0}(\mathbf{x})\right] \\
\psi_{\alpha}^{\beta, 0}(\mathbf{x})=\sum_{l} J_{l}\left(\lambda_{b}\left|\mathbf{x}-\mathbf{x}_{\alpha}\right|\right) e^{i l \theta\left(\mathbf{x}-\mathbf{x}_{\alpha}\right)} \psi_{l, \alpha}^{\beta, 0}
\end{gathered}
$$

$$
\begin{aligned}
& \psi_{l, \alpha}^{M 0}=\frac{\mu_{0} e \omega}{2} e^{i \frac{\omega}{v} x_{\alpha} \pm i \gamma\left(y_{\alpha}-y_{0}\right)}\left( \pm \frac{i}{\lambda_{b}}\right) i^{l} e^{-i l \theta_{K^{ \pm}}} \\
& \psi_{l, \alpha}^{N 0}=\frac{\mu_{0} e \omega}{2} e^{i \frac{\omega}{v} x_{\alpha} \pm i \gamma\left(y_{\alpha}-y_{0}\right)}\left(\frac{k_{z} \omega}{q_{b} v \gamma \lambda_{b}}\right) i^{l} e^{-i l \theta_{K^{ \pm}}}
\end{aligned}
$$

Here, $\theta_{K^{ \pm}}$is the argument of two-dimensional vector $K^{ \pm} \equiv(\omega / v, \pm \gamma)$ for a real $\gamma$. In the case of a pure imaginary $\gamma$ we must define $\theta_{K^{ \pm}}$as

$$
e^{i l \theta_{K^{ \pm}}}=\left(\frac{\omega / v \pm i \gamma}{\lambda_{b}}\right)^{l}
$$

Moreover, the \pm in Eq.(19) and (20) corresponds to the sign of $y_{0}-y_{\alpha}$ and $\psi_{l, \alpha}^{\beta 0}$ is the multi-pole coefficient of the incident wave for cylinder $\alpha$. We should note that the $k_{z}$ integration is involved in Eq.(17). However, since $k_{z}$ is a conserved quantity for the cluster, each cylindrical wave with fixed $k_{z}$ is independently scattered by the cluster.

The incident evanescent wave is multiply scattered in the cluster of the cylinders. By using the multiplescattering method, the induced radiation field is selfconsistently determined as follows ${ }^{14}$ If we focus on cylinder $\alpha$, the incident wave consists of the direct term (Eq.(17)) plus the sum of the induced wave scattered by another cylinder $\alpha^{\prime}$. Therefore, the induced wave from cylinder $\alpha$ is obtained by multiplying the t-matrix of cylinder $\alpha$ to the multi-pole components of the incident wave re-expanded around $\mathbf{x}=\mathbf{x}_{\alpha}$. As a result, the self-consistent induced wave is determined as

$$
\begin{aligned}
& \mathbf{E}^{\text {ind }}(\mathbf{x}, \omega)=\int \frac{d k_{z}}{2 \pi} e^{i k_{z}\left(z-z_{0}\right)}\left[\left(-\frac{1}{\lambda_{b}} \hat{z} \times \nabla\right) \psi^{M, \text { ind }}(\mathbf{x})\right. \\
& \left.+\left(\frac{i k_{z}}{\lambda_{b} q_{b}} \nabla_{\|}+\frac{\lambda_{b}}{q_{b}} \hat{z}\right) \psi^{N, \text { ind }}(\mathbf{x})\right], \\
& \begin{array}{l}
\psi^{\beta, \text { ind }}(\mathbf{x})=\sum_{l, \alpha} H_{l}\left(\lambda_{b}\left|\mathbf{x}-\mathbf{x}_{\alpha}\right|\right) e^{i l \theta\left(\mathbf{x}-\mathbf{x}_{\alpha}\right)} \psi_{l, \alpha}^{\beta, \text { ind }}, \\
\psi_{l, \alpha}^{\beta, \text { ind }}=\sum_{\beta^{\prime}} t_{l, \alpha}^{\beta \beta^{\prime}}\left(\psi_{l, \alpha}^{\beta^{\prime}, 0}+\sum_{l^{\prime}} \sum_{\alpha^{\prime} \neq \alpha} G_{l \alpha, l^{\prime} \alpha^{\prime}} \psi_{l^{\prime}, \alpha^{\prime}}^{\beta^{\prime}, \text { ind }}\right),
\end{array} \\
& G_{l \alpha, l^{\prime} \alpha^{\prime}}=H_{l^{\prime}-l}^{(1)}\left(\lambda_{b} \rho_{\alpha \alpha^{\prime}}\right) e^{i\left(l^{\prime}-l\right) \theta_{\alpha \alpha^{\prime}} .}
\end{aligned}
$$

Here, $G_{l \alpha, l^{\prime} \alpha^{\prime}}$ is the propagator from cylinder $\alpha^{\prime}$ to $\alpha$, and in its expression $\rho_{\alpha \alpha^{\prime}}$ and $\theta_{\alpha \alpha^{\prime}}$ are the magnitude and the argument of $\mathbf{x}_{\alpha}-\mathbf{x}_{\alpha^{\prime}}$, respectively.

As was mentioned in Sec. II, the induced wave contains propagating components with real $\lambda_{b}$, which have a net flux at $\rho \rightarrow \infty$. This implies that a radiation emission takes place when a charged particle passes near the cluster. As a result, the charged particle losses its energy via the emission. In addition, if the cylinders are lossy having positive imaginary part in $\varepsilon_{a}(\omega)$, a part of the energy is absorbed in the cylinders. The total energy loss is then the sum of the radiation emission and the absorption. Qualitatively, the loss can be calculated with the exerted force by the induced field reacting on the particle. Thus, its expression is given by

$$
P_{e l}(\omega)=-\frac{e}{2} \operatorname{Re} \int d t e^{-i \omega t} \mathbf{v} \cdot \mathbf{E}^{\mathrm{ind}}\left(\mathbf{x}_{t}, \omega\right)
$$


per unit angular frequency. The integral over $t$ yields

$$
\begin{aligned}
& P_{e l}(\omega)=\int \frac{d k_{z}}{2 \pi} P_{e l}\left(\omega, k_{z}\right) \\
& P_{e l}\left(\omega, k_{z}\right)=\frac{1}{2} \mu_{0} e^{2} \omega \sum_{l, \alpha} \operatorname{Re}\left[e^{-i \frac{\omega}{v} x_{\alpha} \pm i \gamma\left(y_{0}-y_{\alpha}\right)}\right. \\
& \left.\quad \times(-i)^{l} e^{i l \theta_{K^{ \pm}}}\left( \pm \frac{i}{\lambda_{b}} \psi_{l, \alpha}^{M, \text { ind }}-\frac{k_{z} \omega}{v q_{b} \lambda_{b} \gamma} \psi_{l, \alpha}^{N, \text { ind }}\right)\right]
\end{aligned}
$$

Here, \pm in the above equation is referred as the sign of $y_{0}-y_{\alpha}$.

On the other hand the net flux of the induced radiation emission is obtained by

$$
\begin{aligned}
& P_{e m}(\omega)=\lim _{\rho \rightarrow \infty} \frac{1}{2} \int d z d \theta \rho \\
& \quad \times \operatorname{Re}\left[\left(\mathbf{E}^{\text {ind }}(\mathbf{x}, \omega)\right)^{*} \times \mathbf{H}^{\text {ind }}(\mathbf{x}, \omega)\right] \cdot \hat{\rho} .
\end{aligned}
$$

per unit angular frequency. Using the asymptotic form of the induced field (Eq.(22) ), the net flux turns out to be

$$
\begin{aligned}
& P_{e m}(\omega)=\int_{-q_{b}}^{q_{b}} \frac{d k_{z}}{2 \pi} P_{e m}\left(\omega, k_{z}\right), \\
& P_{e m}\left(\omega, k_{z}\right)=\frac{\mu_{0} e^{2} \omega \lambda_{b}}{8} \int d \theta\left(\left|f^{M}(\theta)\right|^{2}+\left|f^{N}(\theta)\right|^{2}(\beta 2)\right. \\
& f^{\beta}(\theta)=\sqrt{\frac{2}{\pi \lambda_{b}}} \sum_{l, \alpha} e^{-i \lambda_{b} \hat{\rho} \mathbf{x}_{\alpha}+i l \theta}(-i)^{l+1} \psi_{l, \alpha}^{\beta, \text { ind }}
\end{aligned}
$$

The cutoff of the $k_{z}$ integral comes from the fact that the scattered wave becomes evanescent when $\left|k_{z}\right|>q_{b}$. If there is no absorption in the cylinder, the energy loss must be equal to the net flux of the radiation emission, that is,

$$
P_{e l}\left(\omega, k_{z}\right)=P_{e m}\left(\omega, k_{z}\right)
$$

This equality serves as a criterion of the correctness and the convergence of numerically calculated EEL spectrum.

In the case of an isolated cylinder, the integration over $\theta$ in Eq. (32) can be performed analytically, yielding

$$
P_{e m}\left(\omega, k_{z}\right)=\frac{1}{2} \mu_{0} \omega e^{2} \sum_{l \beta}\left|\sum_{\beta^{\prime}} t_{l}^{\beta \beta^{\prime}} \psi_{l}^{\beta^{\prime} 0}\right|^{2} .
$$

First of all, let's consider the EEL in the isolated Aluminum cylinder. The EEL spectrum of the cylinder has a sequence of peaks at the frequencies of the SPP modes. This is because the t-matrix has a SPP pole on the real axis in the complex plane of $\omega$ as can be seen in Eq. (7) and (12). To see the correspondence of the dispersion relation of the SPP, Fig.2 shows the integrand $P_{e l}\left(\omega, k_{z}\right)$ of the EEL spectrum, changing the $k_{z}$ value from 0 to 10 in units of $\omega_{p} / c$. Here, the velocity and the impact parameter of the charged particle were taken to $0.4 c$ and $2 r$, respectively. In addition to the main EEL peak around $\omega=\omega_{p} / \sqrt{2}$, another EEL peak appears much below

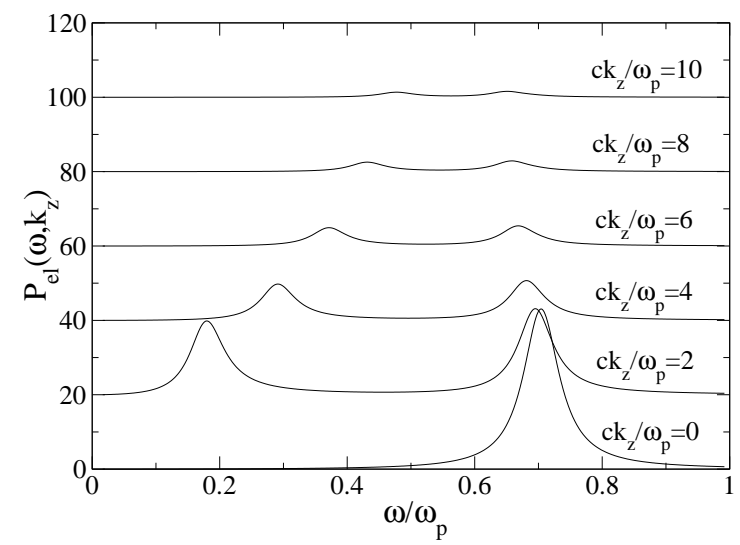

FIG. 2: The electron energy loss spectrum in an isolated Aluminum cylinder with diameter $2.5[\mathrm{~nm}]$ for several $k_{z}$ values are shown. The inverse relaxation time $\eta$ of the Drude dielectric function was taken to be $\hbar \eta=1[\mathrm{eV}]$. The impact parameter, i.e., the distance between the cylindrical axis and $\mathrm{t}$

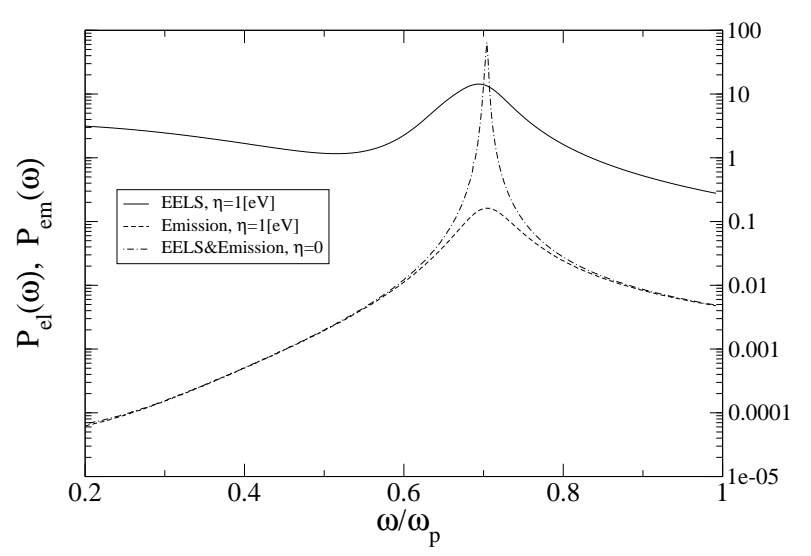

FIG. 3: The integrated EEL and the radiation emission spectra in the isolated Aluminum cylinder. The same parameters as for Fig[2] were used.

$\omega=\omega_{p} / \sqrt{2}$. This peak comes from the SPP mode with $l=0$, as can be understood clearly by comparing with the dispersion relation of the SPP mode with $l=0$. As a general tendency, $P_{e l}\left(\omega, k_{z}\right)$ decreases with increasing $\left|k_{z}\right|$.

Next, we consider how the imaginary part affects the percentage of the radiation emission in the EEL. To this end, we show in Fig 3 the integrated EEL spectrum $P_{e l}(\omega)$ and the integrated radiation emission spectrum $P_{\text {em }}(\omega)$ at $\eta=0,1[\mathrm{eV}]$. At $\eta=0$ these must coincide. As can be seen, when the imaginary part in $\varepsilon_{a}$ is introduced, the radiation emission spectrum is almost unchanged from that in the loss-less cylinder except for the SPP frequency region. However, the EEL spectrum receives a drastic change. According to the figure, the EEL is dominated by the absorption in the cylinder under study. Therefore, the efficiency for converting the kinetic energy of the charged particle to the radiation 


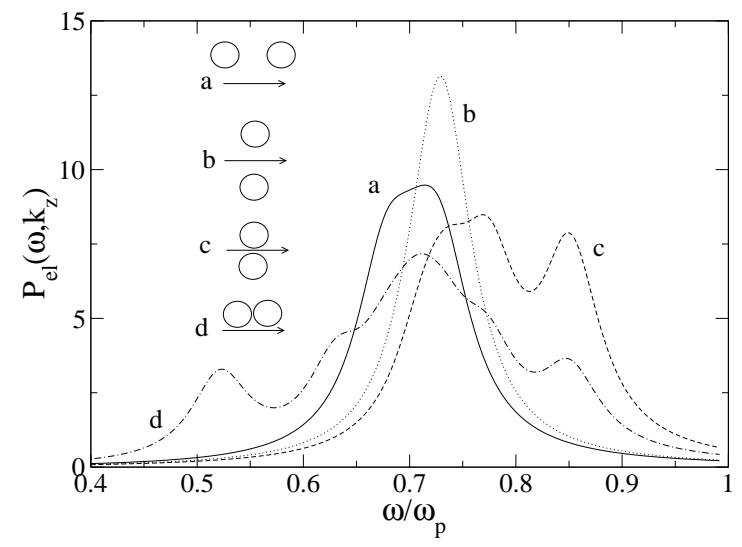

FIG. 4: The EEL spectra in the two identical Aluminum cylinders with various spatial arrangements shown in the insets. $k_{z}=0$ was assumed. See text for the other parameters.

via surface plasmon is very low.

Next, we consider how the spectra change when another cylinder is added. In this case, the multiplescattering of the induced radiation significantly affects the spectra. As we will see, almost touched metallic cylinders causes a drastic change in the spectra as well as in the near-field configuration. This phenomena is closely related to the surface-enhanced Raman scattering $, 30,31.32 .33$ in which the intensity of the induced electro-magnetic field is enhanced more than thousand times as large as that of the incident intensity. As an example, we explored the EEL for various spatial arrangements of the two identical Aluminum cylinders. Fig 4 shows the EEL spectra $P_{e l}\left(\omega, k_{z}\right)$ with $k_{z}=0$ corresponding to the arrangements shown in the insets. In (a) and (b) the two-cylinders are well-separated, the distance between two cylindrical axes being $4 r(r=1.25[\mathrm{~nm}])$, whereas in (c) and (d) they are very close to each other, the distance being $2.16 r$. The distance between the two cylindrical axes and the trajectory of the charged particle is $2 r$ in (a) and (b), and is $1.08 r$ in (c) and (d). As expected, if the two cylinders are well separated along the trajectory (case (a)), the EEL spectrum per cylinder has a single peak near $\omega=\omega_{p} / \sqrt{2}$, though an asymmetry of the peak is observed. In case (b) the two cylinders are separated along the normal direction of the trajectory. This geometry still allows a single peak in the EEL spectrum at $\omega \simeq \omega_{p} / \sqrt{2}$. Since there is the parity symmetry with respect to the trajectory, No electromagnetic modes with the odd parity is involved in case (b). On the other hand, if the two cylinders are very close to each other, several loss peaks appear at the frequencies far from $\omega_{p} / \sqrt{2}$. In particular, the spectrum of the case (d) has two marked peaks at $\omega / \omega_{p} \simeq 0.52$ and 0.84 . The latter peak is shared also by case (c). The peak at $\omega / \omega_{p} \simeq 0.52$ is related to the weakly resonant cavity modes localized in the groove between the two cylinders. To confirm this, we show the field intensity $\left|H_{z}(\mathbf{x})\right|^{2}$ at the peak frequency in Fig $\mathbf{5}$ In the figure

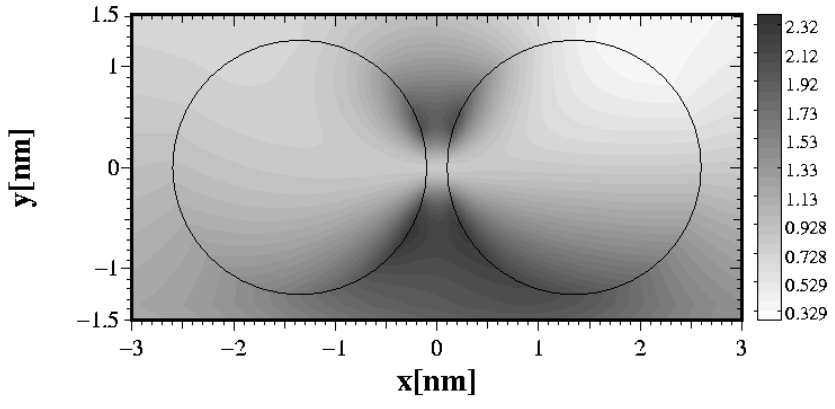

FIG. 5: The field intensity $\left|H_{z}(\mathbf{x})\right|^{2}$ induced by a running charged particle whose velocity is $0.4 c$ is plotted at $\left(k_{z}, \omega\right)=$ $\left(0,0.523 \omega_{p}\right)$, where a peak is observed in the EEL spectrum. The solid line stands for the edges of the two cylinders. The trajectory of the charged particle is at $y=-1.35[\mathrm{~nm}]$.

we can see clearly that the peak is caused by the cavity mode in the groove. As for the peak at $\omega / \omega_{p} \simeq 0.84$ we couldn't find a clear evidence of the resonant cavity mode, as the field intensity at the peak frequency has another local maximum at a boundary of the cylinder beside that in the grooves. This feature suggests that this peak to be caused by a strong mixing of a cavity mode and the SPP modes. If the electron runs across the groove of the almost touched cylinders (case (c)), the peak at $\omega \simeq 0.52 \omega_{p}$ disappears owing to the symmetry mismatch and the peak at $\omega \simeq 0.84 \omega_{p}$ receives an enhancement.

Regarding the radiation emission spectrum in the two cylinders, its features are more or less similar to those in Fig 4 though the magnitude of $P_{e m}\left(\omega, k_{z}\right)$ is much smaller than that of $P_{e l}\left(\omega, k_{z}\right)$ at $k_{z}=0$.

\section{ELECTRON RUNNING OUTSIDE PHOTONIC CRYSTAL}

A photonic crystal that consists of a periodic array of metallic cylinders has a rich spectrum in it, including infinite SPP bands for the TE polarization ${ }^{34.35}$ and a low-frequency cutoff for the TM polarization 36.37 Combining these properties with a running charged particle, the system can react as a novel light emitter. In fact, when a charged particle passes near the photonic crystal, it induces the emission of real photon as was first pointed out by Smith and Purcell for a metallic grating ${ }^{20}$ In the photonic crystal, this phenomena can be interpreted in two ways. One interpretation is as follows. The incident evanescent wave from the charged particle acquires an Umklapp momentum transfer in the photonic crystal, thereby coming into the light cone in the $\left(\omega, k_{\|}\right)$ space, $k_{\|}$being the wave vector parallel to the bound- 


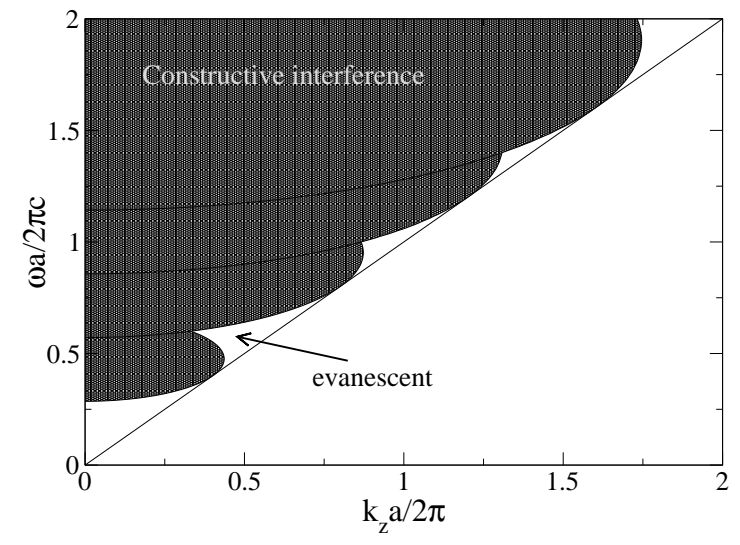

FIG. 6: The phase diagram of the induced radiation emission in a periodic array of cylinders with lattice constant $a$ is shown. The Smith-Purcell radiation is possible only in the shaded region of the $\left(k_{z}, \omega\right)$ plane. The velocity of the charged particle was taken to be $v=0.4 c$. The solid line is the light line.

ary of the photonic crystal. As a result, real photon is emitted from the photonic crystal. The other interpretation is to regard the phenomena as a coherent radiation emission from different cylinders. Though these two interpretations are equivalent, the two points of view give us a deep insight of the Smith-Purcell(SP) radiation in the photonic crystal.

In the case of an isolated cylinder, the induced radiation emission is possible when $q_{b}^{2}>k_{z}^{2}$, i.e., when the emitted light is inside the light cone of the $\left(\omega, k_{z}\right)$ space. On the other hand, the condition that the evanescent light turns out to be a real photon via the Umklapp momentum transfer in the photonic crystal is given by

$$
q_{b}^{2}-k_{z}^{2}-\left(\frac{\omega}{v}-n \frac{2 \pi}{a}\right)^{2}>0,
$$

$a$ being the pitch of the photonic crystal. Therefore, the allowed frequency range of the SP radiation is

$$
\begin{aligned}
& \tilde{\omega}_{-}<\tilde{\omega}<\tilde{\omega}_{+}, \quad{\tilde{k_{z}}}^{2}<\frac{n^{2} \varepsilon_{b}}{\left(\frac{c}{v}\right)^{2}-\varepsilon_{b}}, \\
& \tilde{\omega}_{ \pm}=\frac{n \frac{c}{v} \pm \sqrt{n^{2} \varepsilon_{b}-\left(\left(\frac{c}{v}\right)^{2}-\varepsilon_{b}\right) \tilde{k}_{z}}}{\left(\frac{c}{v}\right)^{2}-\varepsilon_{b}}
\end{aligned}
$$

where $\tilde{\omega}=\omega a / 2 \pi c$ and $\tilde{k_{z}}=k_{z} a / 2 \pi$. If the above condition is not satisfied in the light cone, a destructive interference among the induced radiation fields from different cylinders occurs, leading to the prohibition of the radiation emission. This also implies that if there is no absorption in the photonic crystal, the concerned range in the $\left(\omega, k_{z}\right)$ space does not contribute to the EEL in the photonic crystal. The phase diagram of the radiation emission at $v=0.4 c$ is shown in Fig [ In Fig 6 the $\mathrm{SP}$ radiation is allowed in the shaded regions. The allowed region becomes narrow in the $\left(\omega, k_{z}\right)$ space as the velocity of the particle decreases. Beside, in the allowed region of the SP radiation the photonic density of state is in general singular including the Van Hove singularity. Therefore, we may expect a quite rich spectrum of the SP radiation in the photonic crystals.

The rich spectrum is not limited in the SP radiation. We should mention that the photonic band structure exists also outside the shaded region of Fig [6 As long as the imaginary part in $\epsilon_{r}$ is non-zero, the absorption of the induced radiation is inevitable in the photonic crystal. This causes the EEL outside the shaded region, and the EEL is affected significantly by the photonic band structure therein. Inside the shaded region the EEL consists of the radiation emission and the absorption, and these are independent physical observables.

Like as the case of cluster of cylinders, the EEL and the $\mathrm{SP}$ radiation in the photonic crystals can be treated in a unified framework with the multiple-scattering method on the basis of vector cylindrical waves. Pendry and Martin-Moreno presented for the first time an unified framework in terms of the transfer matrix method to argue the EEL and the SP radiation in photonic crystals ${ }^{12}$, The method is based on a discretization of Maxwell's equation on a spatial mesh in order to obtain the realspace transfer matrix 13 They found that the scattering matrix, which is obtained from the transfer matrix, is directly related to the EEL and SP radiation spectra. Their algorithm is easily adapted to the two-dimensional counterpart 29.38 .39 of the layer Korringa-Kohn-RostokerOhtaka (KKRO) method 40.41.42.43.44.45, which is a generalization of the multiple scattering method to periodic systems. The layer-KKRO method has very high accuracy for the photonic crystal under consideration. We should note that the three-dimensional layer-KKRO method was already used for discussing the EEL and the SP radiation in three-dimensional photonic crystals composed of spheres ${ }^{21.22 .23 .24}$

Let's assume that a running charged particle passes outside a finite-thick photonic crystal composed of a periodic array of the Aluminum cylinders. The particle runs with distance $s$ from the boundary normal to the $\Gamma-X$ direction. The schematic illustration of the system under study is shown in Fig 7 In this case the induced radiation field in the outer region of the photonic crystal including the particle trajectory is given by

$$
\begin{aligned}
& \mathbf{E}^{\text {ind }}(\mathbf{x}, \omega)=\sum_{h} \int \frac{d k_{z}}{2 \pi} e^{i \mathbf{K}_{h}^{-} \cdot \mathbf{x}} Q_{-+}\left(h, h_{0}\right) \epsilon^{+}, \\
& \mathbf{K}_{h}^{ \pm}=\left(k_{x}+h, \pm \gamma_{h}, k_{z}\right) \\
& \frac{\omega}{v}=k_{x}+h_{0} \\
& \gamma_{h}=\sqrt{q_{b}^{2}-\left(k_{x}+h\right)^{2}-k_{z}^{2}}
\end{aligned}
$$

where $Q_{ \pm \pm}\left(h, h^{\prime}\right)$, which has spatial tensor index, is the scattering matrix of the photonic crystal ${ }^{42} k_{x}$ is the Bloch momentum in the irreducible surface Brillouin zone associated with the boundary and $h(=2 \pi \mathbf{Z} / a)$ is a reciprocal lattice vector. In the opposite outer region of 


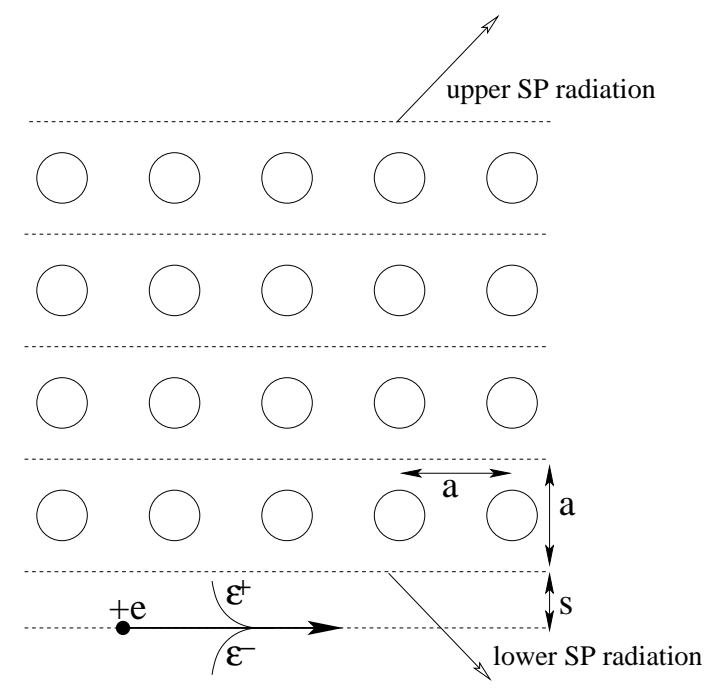

FIG. 7: A charged particle runs with distance $s$ from the boundary of the finite-thick photonic crystal, which is composed of a square array of cylinders with pitch $a$. The polarization vector of the evanescent wave accompanied by the charged particle is denoted by $\epsilon^{ \pm}$.

the photonic crystal the induced radiation field (upper $\mathrm{SP}$ radiation in Fig (7) is given by

$$
\mathbf{E}^{\text {ind }}(\mathbf{x}, \omega)=\sum_{h} \int \frac{d k_{z}}{2 \pi} e^{i \mathbf{K}_{h}^{+} \cdot \mathbf{x}} Q_{++}\left(h, h_{0}\right) \epsilon^{+} .
$$

Quantitatively, the EEL per unit length of the particle trajectory is expressed by the following equation:

$$
\begin{aligned}
& P_{e l}\left(\omega, k_{z}\right)=\frac{1}{4} \mu_{0} e^{2} \omega e^{-2\left|\gamma_{h_{0}}\right| s} \\
& \quad \times\left|\gamma_{h_{0}}\right| \operatorname{Im}\left[\left(\epsilon^{+}\right)^{\dagger} Q_{-+}\left(h_{0}, h_{0}\right) \epsilon^{+}\right] .
\end{aligned}
$$

On the other hand the SP radiation spectrum per unit length is given by

$$
\begin{aligned}
& P_{\text {em }}\left(\omega, k_{z}\right)=\frac{1}{8} \mu_{0} e^{2} \omega e^{-2\left|\gamma_{h_{0}}\right| s} \\
& \times \sum_{h \in \text { open }} \gamma_{h}\left(\left|Q_{++}\left(h, h_{0}\right) \epsilon^{+}\right|^{2}+\left|Q_{-+}\left(h, h_{0}\right) \epsilon^{+}\right|^{2}\right\rangle
\end{aligned}
$$

where the summation is taken over the open diffraction channels.

If the cylinders are loss-less in the photonic crystal, again $P_{e l}\left(\omega, k_{z}\right)=P_{e m}\left(\omega, k_{z}\right)$. This can easily be confirmed by considering flux conservation of through the photonic crystal:

$$
\begin{array}{r}
\sum_{h \in \text { open }} \gamma_{h}\left(\left|Q_{++}\left(h, h_{0}\right) \epsilon^{+}\right|^{2}+\left|Q_{-+}\left(h, h_{0}\right) \epsilon^{+}\right|^{2}\right) \\
=2\left|\gamma_{h_{0}}\right| \operatorname{Im}\left[\left(\epsilon^{+}\right)^{\dagger} Q_{-+}\left(h_{0}, h_{0}\right) \epsilon^{+}\right]
\end{array}
$$

Here, we study two kinds of metallic photonic crystals. One is a dilute photonic crystal that is composed (a)

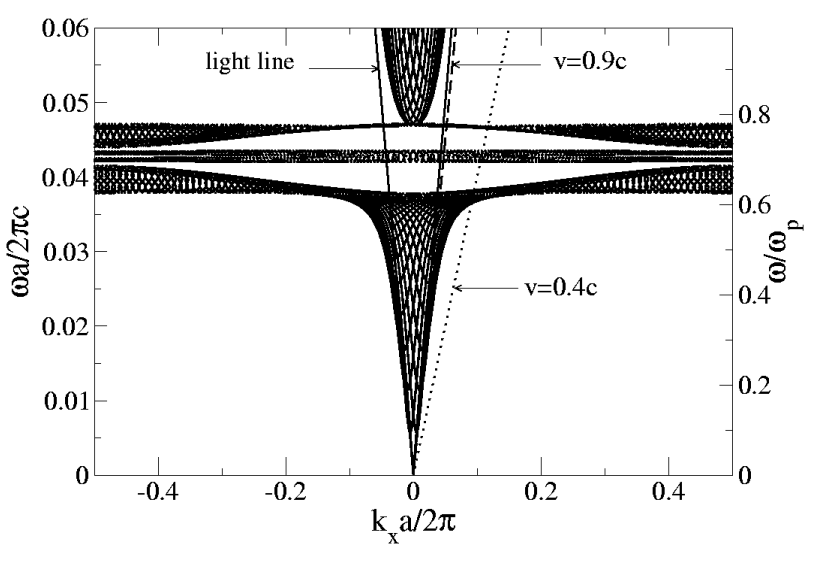

(b)

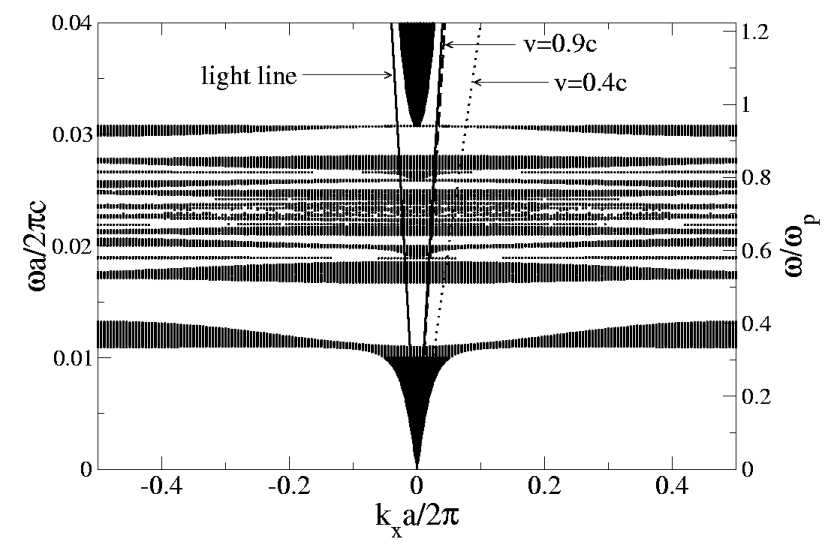

FIG. 8: The photonic band structure of the square lattice of the Aluminum cylinders at $k_{z}=0$ was projected on the surface Brillouin zone associated with the boundary normal to the $\Gamma-X$ direction. In (a) the cylinders are well separated(lattice constant $a=4 r=5[\mathrm{~nm}]$ ), whereas in (b) the cylinders nearly touch $(a=2.16 r=2.7[\mathrm{~nm}])$.

of the square array of the Aluminum cylinders with the lattice constant $a=4 r=5[\mathrm{~nm}]$. The other is its dense version which corresponds to the cases (c) and (d) of the coupled two cylinders treated in the last section $(a=2.16 r=2.7[\mathrm{~nm}])$. The photonic band structures of these photonic crystals at $k_{z}=0$ projected on the surface Brillouin zone associated with the boundary normal to the $\Gamma-X$ direction are shown in Fig 8 . The band structures were calculated by using the two-dimensional layer-KKRO method taking $l_{\max }=5$ and 12 in the dilute and dense photonic crystals, respectively. Here, we dropped the band diagram of the S(TM)-polarization, because it is not relevant to our problem. However, at non-zero $k_{z}$ we must take account of both the $\mathrm{S}$ and $\mathrm{P}$ polarizations.

In both the photonic crystals the plasma frequency of the cylinder is much smaller than the lattice scale, so 
that the photonic band structure is very close to that of the empty lattice at high frequencies. However, below $\omega=\omega_{p}$ many flat bands which characterize the metallic photonic crystals appear. These bands are generally anisotropic, reflecting the $C_{4 v}$ symmetry of the square lattice, and have a singular state density. In the dilute photonic crystal, many bands are concentrated near $\omega=\omega_{p} / \sqrt{2}$. This indicates that they are merely a tightbinding coupling of the SPP of the isolated cylinder. In principle, we can find infinite numbers of the flat bands at $\omega=\omega_{p} / \sqrt{2}$, which are quite difficult to distinguish. On the other hand, in the dense photonic crystal the flat bands are diverse in frequency, whereas their center is still at $\omega=\omega_{p} / \sqrt{2}$. Some of the bands are originated from the cavity mode localized in the groove of the two cylinders. However, most flat bands are considered to be related with the SPP of an Aluminum cylinder.

In Fig 8 the dispersion lines of the charged particle $\left(k_{x}=\omega / v+h_{0}\right)$ at $v=0.4 c$ and $0.9 c$ as well as the light line $\left(\omega=c k_{x}\right)$ were also plotted. In the frequency range concerned only the line with $h_{0}=0$ is relevant, because the threshold of the SP radiation occurring along the Umklapp shifted line of $h_{0}=2 \pi / a$ is rather high ( $\tilde{\omega}_{-} \simeq 0.286$ and 0.474 in Eq. (38) for $v=0.4 c$ and $0.9 c$, respectively). As a result, the EEL is caused solely by the absorption in the frequency range concerned. When the dispersion line meets the shaded region of the projected band diagram, the charged particle can excite an eigenmode in the photonic crystal and thus causes an enhanced absorption loss in EEL. Strictly speaking, the projected band scheme should be used to understand the feature of the photonic crystal with infinite thickness along the $\Gamma-X$ direction. Since we are considering a finite-thick photonic crystal, the shaded region in Fig 8 must be regarded as a set of the dispersion curves of the eigenmodes in the finite-thick photonic crystal. Apparently, as the thickness increases, the dispersion curves fills up with the shaded region.

Fig 9 shows $P_{e l}\left(\omega, k_{z}\right)$ with $k_{z}=0$ of the two photonic crystals, varying number of layers. The velocity of the charged particle was taken to $0.4 c$ and the impact parameter $s$ was taken to zero. Concerning the dilute photonic crystal, the EEL spectrum has the double peaks near $\omega=\omega_{p} / \sqrt{2}$ in the mono-layer case. This feature already appeared in the result of the two separated cylinders (see Fig (4), where an asymmetry of the loss peak is observed. As the number of layers increases, the double peaks disappear and the spectrum converges to a certain function which has single peak near $\omega=\omega_{p} / \sqrt{2}$. The converged spectrum is not so far from the EEL spectrum of the isolated cylinder. These features are consistent with the numerical results on the projected band structure (Fig 8): The dispersion line of the particle with $v=0.4 c$ meets only the flat bands near $\omega=\omega_{p} / \sqrt{2}$. We can infer that the single peak in the EEL spectrum is caused by the broadening of the flat bands owing to the non-zero imaginary part in $\varepsilon_{a}$.

As for the dense photonic crystal, there are several
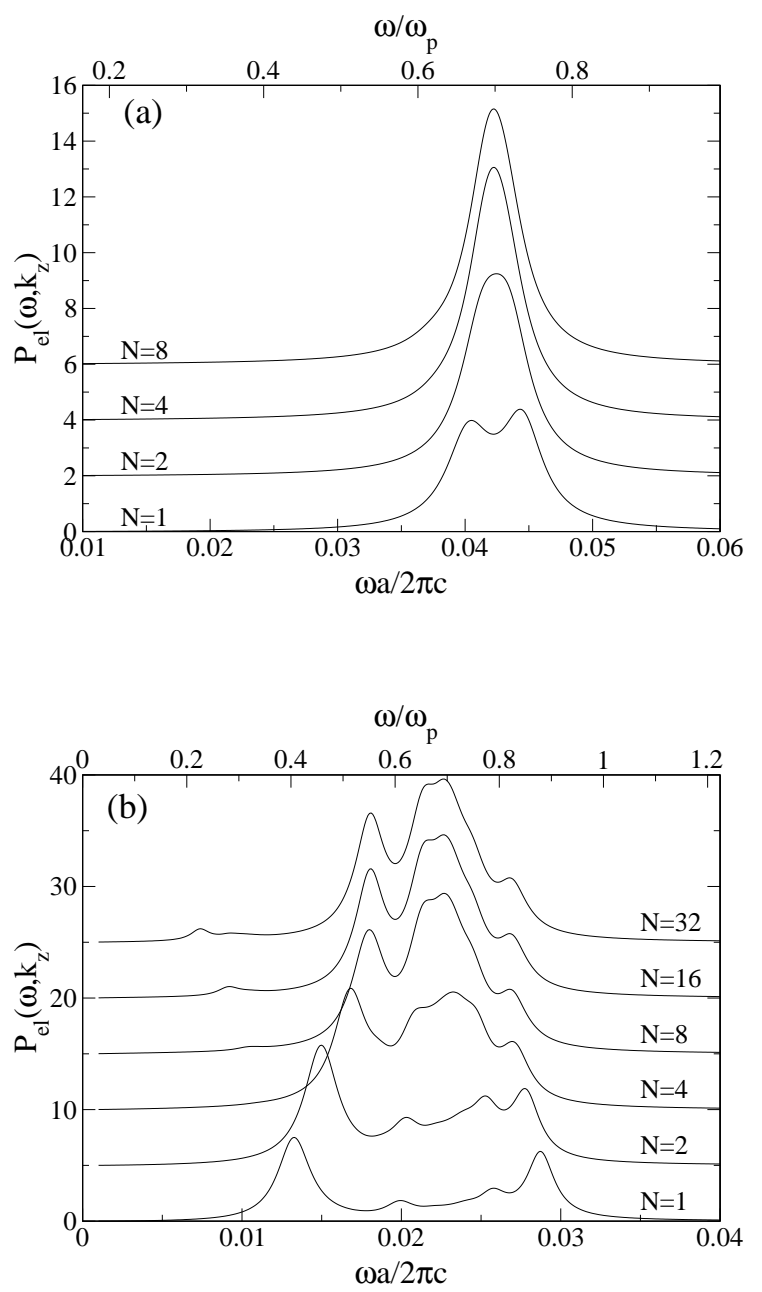

FIG. 9: The EEL spectrum of the photonic crystals at $k_{z}=0$, varying the number of layers. The velocity of the charged particle was taken to $v=0.4 c$. In (a) the cylinders are well separated(lattice constant $a=4 r$ ), whereas in (b) the cylinders nearly touch $(a=2.16 r)$. The trajectory of the charged particle is just on the boundary of the photonic crystal (i.e. $s=0)$.

loss peaks whose positions change as the number of layers increases. Compared with the case of coupled two cylinders, the peak positions of the EEL spectrum in the photonic crystal are well correlated with those of case (d) of Fig (4). In particular, the two peaks at $\omega \simeq 0.55 \omega_{p}$ and $0.82 \omega_{p}$ at $N=32$ are of reminiscences of those in case (d), and the corresponding flat bands, which have relatively large widths in frequency, can be observed in Fig 8 (b). Again, above $\omega a / 2 \pi c \simeq 0.01$ the EEL spectrum converges to a certain function with increasing number of layers, though the convergence progresses slowly compared with that in the dilute photonic crystal. A remarkable feature in this case appears below $\omega a / 2 \pi c=0.01$, where a frequency shift of a small loss peak is observed with increasing $N$. In contrast to the dilute photonic crystal, in such low frequency region the dispersion line $\omega=v k_{x}$ exists within the shaded region of the lowest 


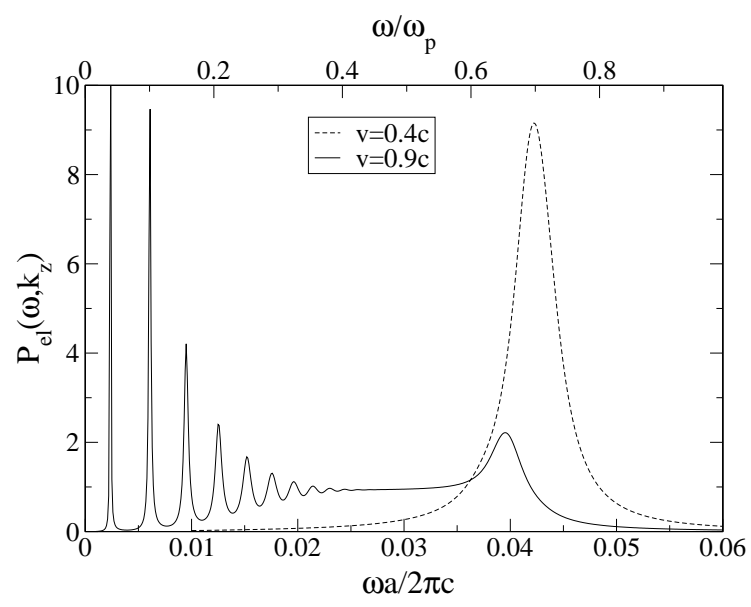

FIG. 10: The EEL spectrum of the dilute photonic crystal having 256 layers at $k_{z}=0$. The velocity of the charged particle was taken to $0.4 c$ (dashed line) and $0.9 c$ (solid line).

band even at $v=0.4 c$. This band does not originate from the SPP modes, and thus the loss peaks found below $\omega a / 2 \pi c$ is different in feature from that by the SPP bands.

The effects of the lowest band in the EEL spectrum can be clearly demonstrated in the dilute photonic crystal with large thickness, using impinging a charged particle impinging with such high-speed that its dispersion line $\omega=v k_{x}$ is in the lowest band in Fig 8 (a). Fig 10 shows the two EEL spectra of $v=0.4 c$ and $0.9 c$ in the dilute photonic crystal having 256 layers. As can be seen in the figure, the lowest band causes very sharp loss peaks whose positions are distributed below $\omega a / 2 \pi c \simeq 0.025$. In order to understand this feature, we must remark that outside the light cone the band becomes the set of the guided modes in the corresponding finite-thick photonic crystal. Moreover, as mentioned later, we can reasonably introduce an effective dielectric function $\varepsilon_{\text {eff }}(\omega)$, which is very close to that of Maxwell-Garnett, $\stackrel{26}{~ t o ~ t h e ~ p h o t o n i c ~}$ crystal under consideration. The effective dielectric function of Maxwell-Garnett is given by

$$
\begin{aligned}
& \varepsilon_{\mathrm{eff}}^{\mathrm{MG}}(\omega)=\varepsilon_{b}\left(1+\frac{2 f \alpha}{1-f \alpha}\right), \\
& \alpha=\frac{\varepsilon_{a}-\varepsilon_{b}}{\varepsilon_{a}+\varepsilon_{b}},
\end{aligned}
$$

$f$ being the filling ration of the cylinders. Using this effective dielectric function, the dispersion relation of the guided modes in the (loss-less) effective medium is determined by

$$
\begin{aligned}
& 1-\left(\frac{-\gamma / \varepsilon_{b}+\gamma^{\prime} / \operatorname{Re}\left(\varepsilon_{\mathrm{eff}}^{\mathrm{MG}}\right)}{\gamma / \varepsilon_{b}+\gamma^{\prime} / \operatorname{Re}\left(\varepsilon_{\mathrm{eff}}^{\mathrm{MG}}\right)}\right)^{2} \exp (2 i \gamma d)=0, \\
& \gamma=\sqrt{\left(\frac{\omega}{c}\right)^{2} \varepsilon_{b}-k_{x}^{2}}, \\
& \gamma^{\prime}=\sqrt{\left(\frac{\omega}{c}\right)^{2} \operatorname{Re}\left(\varepsilon_{\mathrm{eff}}^{\mathrm{MG}}\right)-k_{x}^{2}}
\end{aligned}
$$

$d$ being the thickness of the photonic crystal. By imposing the matching condition of frequency $\omega$ and wave vector $k_{x}(=\omega / v)$, the above equation has a sequence of solutions, which agree with the positions of the sharp loss peaks of $v=0.9 c$ in Fig 10 fairly well.

The convergence of $P_{e l}\left(\omega, k_{z}\right)$ is a direct consequence of the convergence of the scattering matrix $Q_{-+}$itself. As was discussed by Botten et al, the converged value of $Q_{-+}$gives the reflectance of the semi-infinite photonic crystal. ${ }^{39}$ This also implies that using the converged value of $Q_{-+}$, we can extract the effective dielectric function via Fresnel's formula of the interface S-matrix. That is, for the P-polarized incident wave, the scattering matrix $Q_{-+}$of the semi-infinite photonic crystal can be regarded as the interface S-matrix between the background medium and the effective medium:

$$
\begin{aligned}
& {\left[Q_{-+}\left(h_{0}, h_{0}\right)\right]_{p p} \simeq \frac{\gamma / \varepsilon_{b}-\gamma^{\prime} / \varepsilon_{\mathrm{eff}}}{\gamma / \varepsilon_{b}+\gamma^{\prime} / \varepsilon_{\mathrm{eff}}},} \\
& \gamma^{\prime}=\sqrt{\left(\frac{\omega}{c}\right)^{2} \varepsilon_{\mathrm{eff}}-\left(\frac{\omega}{v}\right)^{2}} .
\end{aligned}
$$

Here, $k_{z}=0$ was assumed. The effective dielectric function $\varepsilon_{\text {eff }}$ obtained in this way, along with that of MaxwellGarnett for the dense photonic crystal are shown in Fig[1] The function is not so far from the effective dielectric function of Maxwell-Garnett, though some extra features at $\omega a / 2 \pi c \simeq 0.019$ and 0.026 are observed. In the next section we will see that the EEL spectrum in the photonic crystal, when the charged particle runs inside the photonic crystal, is well reproduced with the effective dielectric function having the extra features. As for the dilute photonic crystal, our effective dielectric function is very close to that of Maxwell-Garnett.

\section{ELECTRON RUNNING INSIDE PHOTONIC CRYSTAL}

When a charged particle runs inside the photonic crystals, the induced radiation field is rather involved owing to the multiple-scattering among the layers above and below the trajectory. However, the scattering matrix formalism is readily adapted to the case as long as the particle does not penetrate any cylinders in the photonic crystals. A schematic illustration of the system under consideration is shown in Fig 12 In this case the induced radiation field reacting back to the charged particle is determined as

$$
\begin{aligned}
& \mathbf{E}^{\mathrm{ind}}(\mathbf{x}, \omega)=\sum_{h}\left(\mathbf{a}_{h} e^{i \mathbf{K}_{h}^{+} \cdot \mathbf{x}}+\mathbf{b}_{h} e^{i \mathbf{K}_{h}^{-} \cdot \mathbf{x}}\right), \\
& \mathbf{a}_{h}=\left(1-Q_{+-}^{d} Q_{-+}^{u}\right)^{-1} Q_{+-}^{d}\left(\epsilon^{-}+Q_{-+}^{u} \epsilon^{+}\right), \\
& \mathbf{b}_{h}=\left(1-Q_{-+}^{u} Q_{+-}^{d}\right)^{-1} Q_{-+}^{u}\left(\epsilon^{+}+Q_{+-}^{d} \epsilon^{-}\right),
\end{aligned}
$$

where $Q_{ \pm \pm}^{u(d)}$ is the scattering matrix of the upper(lower) layers above(below) the trajectory. Beside, the Fourier 

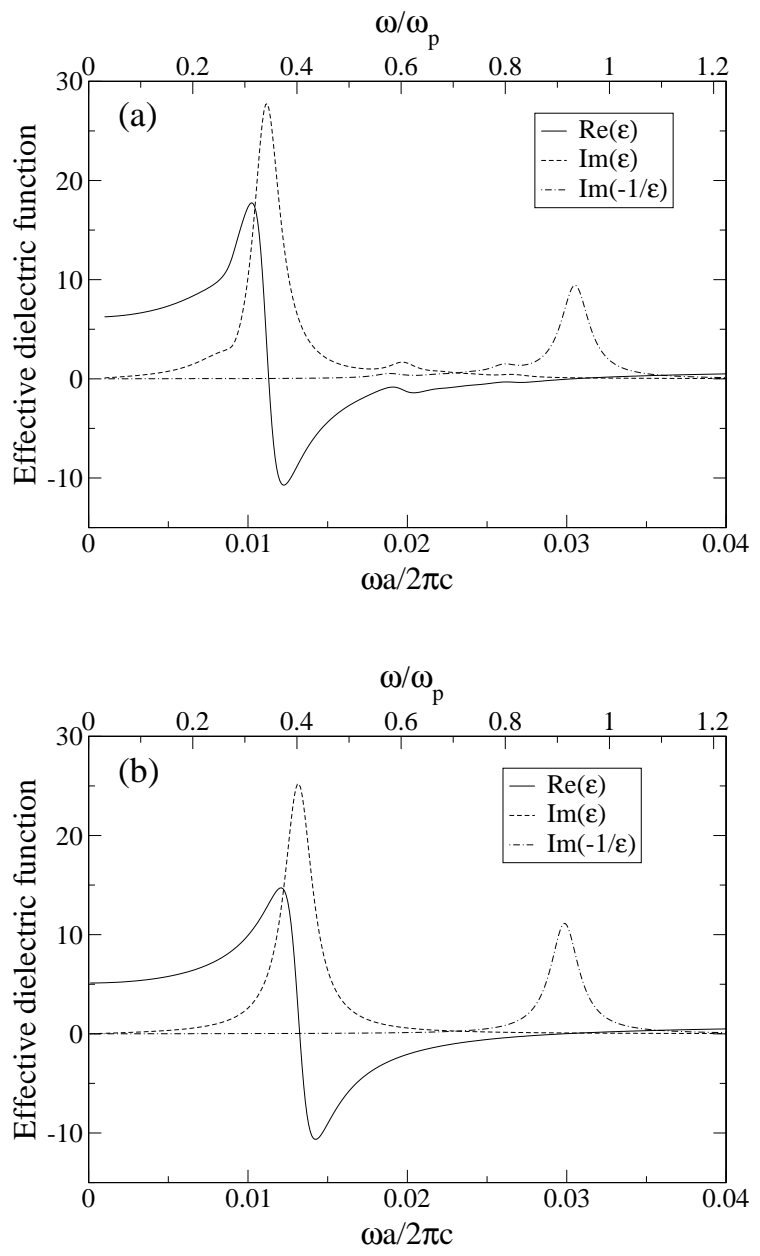

FIG. 11: (a) The effective dielectric function for the Ppolarized light in the dense photonic $\operatorname{crystal}(a=2.16 r)$. The case $k_{z}=0$ was assumed. (b) The effective dielectric function of Maxwell-Garnett in the dense photonic crystal.

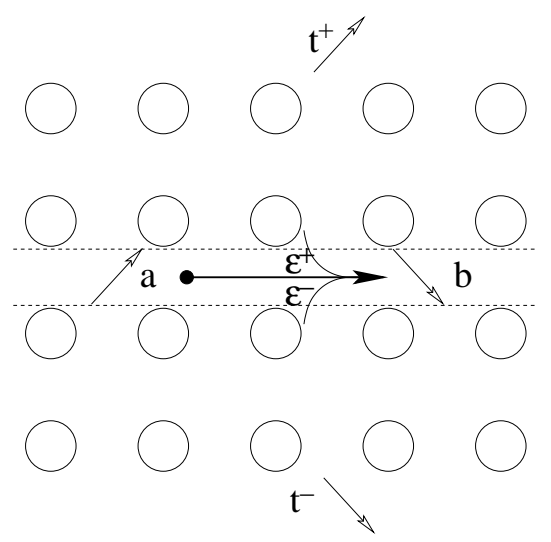

FIG. 12: A charged particle runs inside the photonic crystal with an equal distance from the upper and lower nearest layers of the trajectory. The plane wave coefficients of the induced wave in the void stripe including the trajectory are denoted by $\mathbf{a}$ and $\mathbf{b}$. coefficients of the upper and lower transmitted wave, denoted by $\mathbf{t}_{h}^{ \pm}$, is also obtained as

$$
\begin{aligned}
& \mathbf{t}_{h}^{+}=\sum_{h^{\prime}} Q_{++}^{u}\left(h, h^{\prime}\right)\left(\epsilon^{+} \delta_{h^{\prime} h_{0}}+\mathbf{a}_{h^{\prime}}\right), \\
& \mathbf{t}_{h}^{-}=\sum_{h^{\prime}} Q_{--}^{d}\left(h, h^{\prime}\right)\left(\epsilon^{-} \delta_{h^{\prime} h_{0}}+\mathbf{b}_{h^{\prime}}\right) .
\end{aligned}
$$

Therefore, the EEL and SP radiation spectra per unit length becomes

$$
\begin{aligned}
& P_{e l}\left(\omega, k_{z}\right)=\frac{1}{4} \mu_{0} e^{2} \omega \mid \gamma_{h_{0}} \operatorname{Im}\left(\mathbf{a}_{h_{0}}^{*} \cdot \epsilon^{-}-\mathbf{b}_{h_{0}} \cdot\left(\epsilon^{+}\right)(\$ 9)\right. \\
& P_{s p}\left(\omega, k_{z}\right)=\frac{1}{8} \mu_{0} e^{2} \omega \sum_{h \in \text { open }} \gamma_{h}\left(\left|\mathbf{t}_{h}^{+}\right|^{2}+\left|\mathbf{t}_{h}^{-}\right|^{2}\right) .
\end{aligned}
$$

Again, the flux conservation in a loss-less photonic crystal leads

$$
\begin{aligned}
& \sum_{h \in \text { open }} \gamma_{h}\left(\left|\mathbf{t}_{h}^{+}\right|^{2}+\left|\mathbf{t}_{h}^{-}\right|^{2}\right) \\
& =-2\left|\gamma_{h_{0}}\right| \operatorname{Im}\left(\mathbf{a}_{h_{0}}^{*} \cdot \epsilon^{-}-\mathbf{b}_{h_{0}} \cdot\left(\epsilon^{+}\right)^{*}\right),
\end{aligned}
$$

which implies that the EEL is equal to the SP radiation emission.

At low frequencies we may expect that the EEL in a photonic crystal is somehow approximated by that of a lossy effective homogeneous medium. As is known well, the relativistic EEL in such a medium with permittivity $\varepsilon_{\text {eff }}$ is given by

$$
P_{e l}\left(\omega, k_{z}\right)=\frac{1}{4} \mu_{0} e^{2} \omega \operatorname{Re}\left[\frac{1}{\gamma}\left(1-\left(\frac{c}{v}\right)^{2} \frac{1}{\varepsilon_{\text {eff }}}\right)\right],
$$

per unit length. When $\varepsilon_{\text {eff }}$ is real and the condition $v>c / \sqrt{\varepsilon}$ is satisfied, the above equation is equal to the Cerenkov loss. Otherwise, Eq.(62) can be regarded as the EEL by the absorption. In a homogeneous metal the bulk plasmon dominantly contributes to the EEL, because of the factor $1 / \varepsilon_{\text {eff }}$.

Fig 13 shows the EEL spectra of the dense photonic crystal and its simulation using the effective dielectric function obtained by Eqs (52) and (53). Here, the charged particle runs with velocity $0.4 c$ between the 32 th and 33th layer of the 64-layer thick slab of the dense photonic crystal.

One can observe that the frequency of the main loss peak is larger than $\omega=0.9 \omega_{p}$, which is close to the bulk plasmon frequency. This is quite reasonable, taking account that the dense photonic crystal has a large filling ratio $(\simeq 67 \%)$ and thus is close to the bulk metal of Aluminum. Beside the main loss peak, two small peaks are observed in the EEL spectrum. Using the homogeneous medium approximation with the effective dielectric function obtained in the previous section the above features are well reproduced. It should be emphasized that the small two peaks in the dense photonic crystal can not be explained with the effective medium theory of MaxwellGarnett based on $\varepsilon_{\text {eff }}^{\mathrm{MG}}$ given by Eq. (47). 


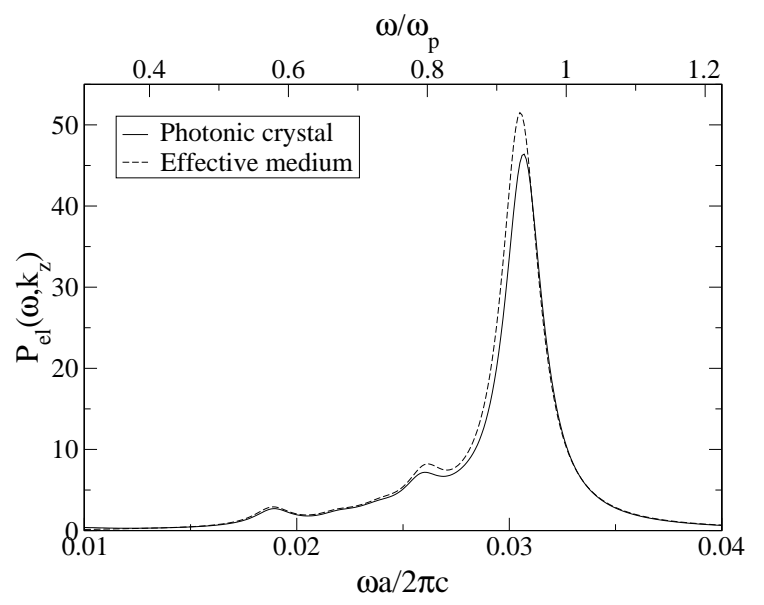

FIG. 13: The EEL spectrum in the dense photonic crystal having 64 layers along the $\Gamma-X$ direction is shown. The EEL spectrum in the effective medium whose dielectric function is given by Fig 11 is also plotted. The velocity of the charged particle is taken to $0.4 c$.

Regarding the EEL spectrum in the dilute photonic crystal, our effective dielectric function as well as the Maxwell-Garnett approximation reproduce the spectrum having a single peak near $\omega=\omega_{p} / \sqrt{2}$ fairly well.

\section{SUMMARY}

In this paper we have presented a fully-relativistic analysis of the EEL and the induced radiation emission in various spatial arrangements of metallic cylinders by using the multiple scattering method and the layer-KKRO method. In an isolated metallic cylinder with a nanoscale diameter we showed that the EEL is dominated by the absorption rather than the induced radiation emission. Thus, the efficiency of converting the kinetic energy of the charged particle to the radiation emission is very low. In the two identical metallic cylinders a variety of EEL peaks appear. Some of them are attributed to the cavity mode localized in the groove between the cylinders. Such a cavity mode as well as the SPP modes become the seed of the flat bands in a dense periodic arrangement of the metallic cylinders. After presenting a mathematical description of the EEL and the SP radiation emission in two-dimensional photonic crystals composed of cylin- ders, we showed the numerical results of the EEL spectra in both dilute and dense periodic arrays of the metallic cylinders. In the dilute photonic crystal the EEL spectrum has a simple structure. The spectrum has a single peak near $\omega=\omega_{p} / \sqrt{2}$ and is not so far from the EEL spectrum in the isolated cylinder. However, when a high speed charged particle passes near the photonic crystal, a sequence of very sharp loss peaks, which comes from the lowest photonic band guided in the finite-thick photonic crystal, are observed. The peaks are comparable in magnitude with that by the SPP bands. On the other hand in the dense photonic crystal the EEL spectrum is very complicated reflecting the photonic band structure, though a good correspondence to the EEL spectrum in the almost touched two cylinders is observed. In both the photonic crystals the effective dielectric functions, which are obtained with the reflectance of the corresponding semi-infinite photonic crystals, fairly reproduce the EEL spectra when the charged particle runs inside the photonic crystals.

In this paper we have restricted ourselves to various arrays of Aluminum cylinders with a diameter of a few nanometers, bearing carbon nano-tube arrays in mind. Since the plasma wavelength of Aluminum is much larger than the above scale, a metallic photonic crystal composed of the cylinders behaves as if it has an effective dielectric function reflecting the coupled SPPs, in the frequency range concerned. As a result, the SP radiation from the photonic crystal is completely absent. However, it is of great importance to study the EEL and the SP radiation emission spectra when the lattice constant is comparable with or larger than the plasma wavelength of the constituent cylinders. In this case the flat bands of coupled SPPs appear in $\omega a / 2 \pi c \simeq O(1)$, so that the EEL is caused both by the absorption and by the SP radiation. How these bands as well as usual photonic bands affects these spectra is the main theme of the paper II.

\section{Acknowledgments}

The authors would like to thank J. Inoue and S. Yamaguti of Chiba University for useful comments. This work was supported by "Promotion of Science and Technology" from the Ministry of Education, Sports, Culture, Science and Technology of Japan.
1 U. Kreibig and M. Vollmer, Optical properties of metal clusters (Springer-Verlag, Berlin, 1995).

2 J.D. Joannopoulos, R.D. Meade, and J.N. Winn, Photonic Crystals (Princeton University Press, Princeton, 1995).

${ }^{3}$ K. Sakoda, Optical Properties of Photonic Crystals, (Springer-Verlag, Berlin, 2001).

4 V.G. Veselago, Sov. Phys. Usp. 10, 509 (1968).

5 J. B. Pendry, Phys. Rev. Lett. 85, 3966 (2000).
${ }^{6}$ R.A. Shelby, D.R. Smith, and S. Shultz, Science 292, 77 (2001).

7 A.A. Lucas, L. Hernard, and Ph. Lambin, Phys. Rev. B 49, 2888 (1994).

8 R.H. Ritchie and A. Howie, Philos. Mag. A 58, 753 (1988).

9 M. Schmeits, Phys. Rev. B 39, 7567 (1988).

10 J.M. Pitarke and A. Rivacoba, Surf. Sci. 377-379, 294 (1997). 
11 G.F. Bertsch, H. Esbensen, and B.W. Reed, Phys. Rev. B 58, 14031 (1998).

12 J.B. Pendry and L. Martin-Moreno, Phys. Rev. B 50, 5062 (1994).

13 J.B. Pendry and A. MacKinnon. Phys. Rev. Lett. 69, 2772 (1992).

14 F.J. García de Abajo, Phys. Rev. Lett. 82, 2776 (1999).

15 F.J. García de Abajo, Phys. Rev. B 60, 6103 (1999).

16 F.J. García de Abajo and A. Howie, Phys. Rev. Lett. 80, 5180 (1998).

17 F.J. García de Abajo and A. Howie, Phys. Rev. B 65, 115418 (2002).

18 F.J. García-Vidal and J.M. Pitarke, Eur. Phys. J. B 22, 257 (2001).

19 N. Yamamoto, K. Araya, and F.J. García de Abajo, Phys. Rev. B 64, 205419 (2001).

20 S.J. Smith and E. M. Purcell, Phys. Rev. 92, 1069 (1953).

21 F.J. García de Abajo, Phys. Rev. E 61, 5743 (2000).

${ }^{22}$ K. Ohtaka and S. Yamaguti, Opt. Spectrosc. 91, 477 (2001).

23 S. Yamaguti, J. Inoue, and K. Ohtaka, Phys. Rev. B 66, 085209 (2002).

24 S. Yamaguti, J. Inoue, O. Haeberlé and K. Ohtaka, Phys. Rev. B 66, 195202 (2002).

${ }^{25}$ L.D. Landau, E.M. Lifshitz, and L.P. Pitaevskii, Electrodynamics of Continuous Media (Pergamon Press, Oxford, 1984).

26 J.C. Maxwell-Garnett, Philos. Trans. R. Soc. London A 203, 385 (1904).

27 J.M. Pitarke, F.J. García-Vidal, and J.B. Pendry, Phys. Rev. B 57, 15261 (1998).

28 D.R. Smith, S. Shultz, P. Markos, and C.M. Soukoulis,
Phys. Rev. B 65, 195104 (2002).

29 K. Ohtaka, T. Ueta, and K. Amemiya, Phys. Rev. B 57, 2550 (1998).

30 M. Inoue and K. Ohtaka, Phys. Rev. B 26, 3487 (1982).

31 M. Inoue and K. Ohtaka, J. Phys. Soc. Jpn 52, 1457 (1983).

32 M. Inoue and K. Ohtaka, J. Phys. Soc. Jpn 52, 3853 (1983).

33 F.J. García-Vidal and J. B. Pendry. Phys. Rev. Lett. 77, 1163 (1997).

34 T. Ito and K. Sakoda, Phys. Rev. B 64, 045117 (2001).

35 T. Ochiai and J. Sánchez-Dehesa, Phys. Rev. B 65, 245111 (2002).

36 V. Kuzmiak, A.A. Maradudin, and F. Pincemin, Phys. Rev. B 50, 16835 (1994).

37 J.B. Pendry, A.J. Holden, W.J. Stewart, and I. Youngs, Phys. Rev. Lett. 76, 4773 (1996)

${ }^{38}$ K. Ohtaka and H. Numata, Phys. Lett. 73A, 411 (1979).

39 L.C. Botten, N.A. Nicorovici, R.C. McPhedran, C. Martijn de Sterke, and A.A. Asatryan, Phys. Rev. E 64, 046603 (2001).

40 J. Korringa, Physica 13, 392 (1947).

41 W. Kohn and N. Rostoker, Phys. Rev. 941111 (1954).

${ }^{42}$ K. Ohtaka, Phys. Rev. B 19, 5057 (1979).

43 K. Ohtaka, J. Phys. C 13, 667 (1980).

44 A. Modinos, Physica A 141, 575 (1987).

45 N. Stefanou, V. Karathanos, and A. Modinos, J. Phys. Condens. Matter 4, 7389 (1992).

46 F.J. García de Abajo and L. A. Blanco, Phys. Rev. B 67, 125108 (2003). 